Pacific Journal of Mathematics

GENERIC PROPERTIES OF THE ADJUCTION MAPPING FOR 


\title{
GENERIC PROPERTIES \\ OF THE ADJUNCTION MAPPING FOR SINGULAR SURFACES AND APPLICATIONS
}

\author{
Marco Andreatta, Mauro Beltrametti, \\ AND ANDREW J. SOMMESE
}

\begin{abstract}
In the last years many new results on the classical problem of classifying smooth surfaces in the projective space in terms of their extrinsic projective and intrinsic geometric invariants have been made by using the adjunction mapping. In this paper we extend the existence theorem for the adjunction mapping to the case of singular surfaces. Although the mapping is only meromorphic we obtain many inequalities known previously only in the smooth case. As an illustration of the results we given a very complete answer in the singular case, parallel to the smooth result, to the question of when a singular surface can "have a hyperelliptic hyperplane section".
\end{abstract}

Introduction. In modern times Sommese [S1] introduced the adjunction mapping to attack this problem. This line of attack led to a complete solution of this question in [S1], [V], [S2], [E], [Se], [S-V]. The methods introduced have had wide applicability to the solution of classification questions for projective manifolds; see [S5], [S-V] for applications and references.

We are interested in how much of the smooth technique survives when no smoothness assumptions are made. As a test we pose and give a surprisingly complete answer to the following problem.

Problem. Let $\Sigma$ be an irreducible complex surface embedded in some complex projective space $\mathbb{P}^{r}$ and $\eta: S \rightarrow \Sigma$ be the normalization of $\Sigma$ with $L$ the pullback of $S$ under $\eta$ of $\mathscr{O}_{\mathrm{pr}}(1)$. If there is a smooth hyperelliptic curve $C \in|L|$, the linear system associated to $L$, then describe $(S, L)$.

The main tool we use is the meromorphic map associated to $\Gamma\left(K_{S} \otimes L\right)$, which is called the adjunction map. In [A-S1], the first and last author showed that if $\pi: S^{\prime} \rightarrow S$ is the minimal desingularization of $S$, then $K_{S^{\prime}} \otimes L^{\prime}, L^{\prime}=\pi^{*} L$, is nef and big except when $\left(S^{\prime}, L^{\prime}\right)$ and $(S, L)$ are of very restricted type. In the case when $K_{S^{\prime}} \otimes L^{\prime}$ is nef, $h^{0}\left(K_{S^{\prime}} \otimes L^{\prime}\right) \neq 0$ (see $\left.(0.6),(0.7)\right)$. These results allow us to 
prove many inequalities between invariants which are standard consequences of the adjunction mapping in the smooth case. The plan of this paper is as follows.

In $\S 0$ we give background material and prove a few new results.

In $\S 1$ we use Reider's theorem ([B], [R]), following Beauville to show that the adjunction map is birational if $K_{S^{\prime}} \otimes L^{\prime}$ is nef with either $c_{1}(L)^{2} \geq 10$ or $c_{1}(L)^{2} \geq 9$ and $L^{\prime} \sim 3 D$ for some effective divisor $D$ such that $D \cdot D=1$. The analogue of this for a smooth $S$ goes back to Van de Ven [V].

In $\S 2$ we prove a number of inequalities that are standard tools in the smooth theory [S1]. E.g., if the Kodaira dimension of $S^{\prime}$ is nonnegative and $c_{1}(L)^{2} \geq 10$, then (see 2.1)

$$
c_{1}\left(K_{S^{\prime}}\right)^{2}+2 g(L) \geq d+2\left(p_{g}\left(S^{\prime}\right)-q\left(S^{\prime}\right)\right)
$$

where $p_{g}\left(S^{\prime}\right)=h^{2,0}\left(S^{\prime}\right)$ and $q\left(S^{\prime}\right)=h^{1,0}\left(S^{\prime}\right)$ are the geometric genus and the irregularity of $S^{\prime}$ and $g(L)$ is the sectional genus of $L$.

In $\S 3$ we give some simple applications. For the main one we derive a result, on when $|L|$ contains even one smooth hyperelliptic curve, that generalizes results of Sommese [S1] and Van de Van [V]. The result is the following (see (3.1)).

THEOREM. With the notation as above, let $L$ be an ample and spanned line bundle on a normal surface $S$. Further assume that $\Gamma(L)$ gives a generically one-to-one map and either $c_{1}(L)^{2} \geq 10$ or $c_{1}(L)^{2} \geq 9$ and $L^{\prime} \sim 3 D, D$ effective divisor with $D \cdot D=1$. If there exists a smooth hyperelliptic curve $C \in|L|$ then $p_{g}\left(S^{\prime}\right)=0, d=c_{1}(L)^{2} \geq g(L)+2$ and either

(i) $q\left(S^{\prime}\right)>0, h^{0}(L)=4, g(L)+2 \geq 3 q\left(S^{\prime}\right)+d / 3$ and there exist at most finitely many smooth hyperelliptic curves in $|L|$;

(ii) $(S, L)$ is a cone or a scroll; or

(iii) $\left(S^{\prime}, L^{\prime}\right)$ is a conic bundle over a smooth curve.

0. Background material. We work over the complex number field $\mathbb{C}$. By variety we mean an irreducible and reduced projective scheme $X$ of dimension $n$. We denote its structure sheaf by $\mathscr{O}_{X}$. For any coherent sheaf $\mathscr{Y}$ on $X, h^{i}(\mathscr{Y})$ denotes the complex dimension of $H^{i}(X, \mathscr{Y})$.

If $X$ is normal, the canonical sheaf $K_{X}$ is defined to be $j_{*} K_{\mathrm{Reg}}(X)$ where $j: \operatorname{Reg}(X) \rightarrow X$ is the inclusion of the smooth points of $X$ and $K_{\operatorname{Reg}(X)}$ is the canonical sheaf of the holomorphic $n$-forms. Note that $K_{X}$ is a line bundle if $X$ is Gorenstein. 
Let $\mathscr{L}$ be a line bundle on $X . \mathscr{L}$ is said to be numerically effective, nef for short, if $\mathscr{L} \cdot C \geq 0$ for each irreducible curve $C$ on $S$, and in this case $\mathscr{L}$ is said to be big if $c_{1}(\mathscr{L})^{n}>0$, where $c_{1}(\mathscr{L})$ is the first Chern class of $\mathscr{L}$. We shall denote by $|\mathscr{L}|$ the complete linear system associated to $\mathscr{L}$ and by $\Gamma(\mathscr{L})$ the space of its global sections. We say that $\mathscr{L}$ is spanned if it is spanned by $\Gamma(\mathscr{L})$.

(0.1) We fix some more notation.

$\sim($ resp. $\approx)$ the numerical (resp. linear) equivalence of divisors;

$\chi(\mathscr{L})=\sum(-1)^{i} h^{i}(\mathscr{L})$, the Euler characteristic of a line bundle $\mathscr{L}$;

$\kappa(X)$, the Kodaira dimension of $X$, that is the Kodaira dimension of a nonsingular model of $X$.

Abuses. Line bundles and divisors are used with little or no distinction. Hence we shall freely switch from the multiplicative to the additive notation and vice versa.

(0.2) Throughout this paper, $S$ always denotes an irreducible projective normal surface. Let $\pi: S^{\prime} \rightarrow S$ be the minimal desingularization of $S$, i.e. $S^{\prime}$ is the unique desingularization of $S$ which is minimal in the sense that the fibres of $\pi$ contain no smooth rational curves $C$ satisfying $C^{2}=-1$. If $L$ is a line bundle on $S$ we will denote by $L^{\prime}$ the inverse image, $\pi^{*} L$. We shall briefly say that $\left(S^{\prime}, L^{\prime}\right)$ is the minimal desingularization of the pair $(S, L)$. If $D$ is a (Weil) divisor we will denote by $D^{\prime}$ the proper transform of $D$. For every Weil divisor $D$ and line bundle $L$ on $S$ the intersection $L \cdot \mathscr{O}_{S}(D)=L \cdot D=L^{\prime} \cdot D^{\prime}$ is well defined.

(0.3) Let $\pi: S^{\prime} \rightarrow S$ be the minimal resolution of the singularities of $S$ and let $\Delta=\pi^{-1}(\operatorname{Irr}(S))$, where $\operatorname{Irr}(S)$ denotes the irrational locus of $S$. We say that $(S, L)$ is a-minimal if there are no smooth rational curves $E$ on $S^{\prime}-\Delta$, with $E \cdot E=-1$ and $\pi^{*} L \cdot E=0$. Note that the pair $\left(S^{\prime}, L^{\prime}\right)$ in $(0.2)$ is clearly $a$-minimal if $L$ is ample; this allows us to apply to $\left(S^{\prime}, L^{\prime}\right)$ the results of $[\mathrm{A}-\mathrm{S} 1]$.

(0.4) The genus formula. Let $L$ be a nef and big line bundle on a normal surface $S$. Then the sectional genus, $g(L)$, of $L$ is defined by the equality $2 g(L)-2=\left(K_{S}+L\right) \cdot L$.

It can be easily seen that $g(L)$ is an integer. Furthermore if there exists an irreducible reduced curve $C$ in $|L|, g(L)$ is simply the arithmetic genus $p_{a}(C)=1-\chi\left(\mathscr{O}_{C}\right)$ of $C$. Note also that $g(L)=g\left(L^{\prime}\right)$, where $\left(S^{\prime}, L^{\prime}\right)$ is the minimal desingularization of $(S, L)$. 
(0.5) Let $S$ be a normal surface and let $L$ be a nef and big line bundle on $S$. We say that the (generically) polarized pair $(S, L)$ is geometrically ruled if $S$ is a $\mathbb{P}^{1}$-bundle, $p: S \rightarrow R$, over a nonsingular curve $R$ and the restriction $L_{f}$ of $L$ to a fibre $f$ of $p$ is $\mathscr{O}_{f}(1)$. We say that $(S, L)$ is a scroll (resp. a conic bundle) over a nonsingular curve $R$ if there is a surjective morphism with connected fibres $p: S \rightarrow R$, with the property that $L$ is relatively ample with respect to $p$ and there exist some $k>0$ and some very ample line bundle $M$ on $R$ such that $\left(K_{S} \otimes L^{2}\right)^{k} \approx p^{*} M$ (resp. $\left.\left(K_{S} \otimes L\right)^{k} \approx p^{*} M\right)$; here $K_{S}^{k}=\left(K_{S}^{\otimes k}\right)^{* *}$.

The following result will be used several times through the paper.

(0.6) LEMma. Let $S$ be a nonsingular surface and let $L$ be a nef and big line bundle on $S$. Assume $(S, L)$ is a-minimal. Then the following are equivalent

(0.6.1) $h^{0}\left(K_{S}+L\right) \neq 0$

$(0.6 .2) h^{0}\left(\left(K_{S}+L\right)^{N}\right) \neq 0$ for some $N>0$;

(0.6.3) $K_{S}+L$ is nef;

$(0.6 .4) g(L) \geq 1$ and $\left(K_{S}+L\right)^{2} \geq 0$.

Proof. The equivalence between (0.6.2) and (0.6.3) is proved in [A-S1], (2.5), while $(0.6 .1) \Rightarrow(0.6 .2)$ and $(0.6 .3) \Rightarrow(0.6 .4)$ are clear. So let us prove that $(0.6 .4)$ implies $(0.6 .1)$. Now we have

$$
H^{0}\left(K_{S}+L\right)=\chi\left(K_{S}+L\right)=g(L)-1+\chi\left(\mathscr{O}_{S}\right) .
$$

Hence if $(0.6 .1)$ would be false, then $\chi\left(\mathscr{O}_{S}\right) \leq 0$. Let $g(L)=1$. Therefore $\left(K_{S}+L\right) \cdot L=0$ and the Hodge index theorem combined with $\left(K_{S}+L\right)^{2} \geq 0$ gives $K_{S} \sim-L$, whence $\chi\left(\mathscr{O}_{S}\right)>0$, a contradiction. So $g(L)>1$ and $\chi\left(\mathscr{O}_{S}\right)<0$. It thus follows that $S$ is ruled; further we claim that $g(L)=q(S)$. Indeed, since $p_{g}(S)=0$, we have

$$
0=h^{0}\left(K_{S}+L\right)=\chi\left(K_{S}+L\right)=\chi\left(L^{-1}\right)=g(L)-q(S) .
$$

Let $d=L \cdot L$. Then the assumption $\left(K_{S}+L\right)^{2} \geq 0$ and genus formula $(0.4)$ yield

$$
K_{S}^{2}+4 g(L)-4 \geq d .
$$

Therefore, since $S$ is ruled and $g(L)=q(S)>1$,

$$
d \leq 8(1-q(S))+4 q(S)-4=4-4 q(S)
$$

a contradiction. 
(0.7) Corollary. Let $S$ be a normal surface, $L$ an ample line bundle on $S$ and let $\left(S^{\prime}, L^{\prime}\right)$ be the minimal desingularization of $(S, L)$. then $K_{S^{\prime}}+L^{\prime}$ is nef if and only if $h^{0}\left(K_{S}+L\right)>\operatorname{length}\left(K_{S} / \mathscr{H}_{S}\right)$, where $\mathscr{K}_{S}$ denotes the Grauert-Riemenschneider canonical sheaf.

Proof. Look at the exact sequence

$$
0 \rightarrow \mathscr{K}_{S} \otimes L \rightarrow K_{S} \otimes L \rightarrow \mathscr{S} \otimes L \rightarrow 0
$$

and note $H^{1}\left(\mathscr{K}_{S} \otimes L\right)=(0)$ by the Grauert-Riemenschneider vanishing theorem and $h^{0}(\mathscr{S} \otimes L)=\operatorname{length}\left(K_{S} / \mathscr{K}_{S}\right)$. Now the statement is an immediate consequence of Lemma (0.6).

In Section 1 we shall use Reider's result for separating general points in the following form

(0.8) THEOREM (Reider, [R]). Let $L$ be a nef and big line bundle on a smooth surface $S$. If $L \cdot L \geq 9$ and the map associated to $\Gamma\left(K_{S}+L\right)$ is not a birational morphism, then there exists an effective divisor $D$ on $S$ such that

$$
\begin{array}{ll}
L \cdot D=0, & D^{2}=-1 ; \\
L \cdot D=0, & D^{2}=-1 \text { or } 0 \\
L \cdot D=2, & D^{2}=0 ; \text { or } \\
L \sim 3 D, & D^{2}=1 .
\end{array}
$$

(0.9) Castelnuovo's bound. Let $X$ be an $n$-dimensional normal variety and let $L$ be a big and spanned line bundle on $X$. Further assume that the map $\varphi: X \rightarrow \mathbb{P}^{N}$ associated to $\Gamma(L)$ is generically one to one. Let $C$ be a smooth curve obtained as transversal intersection of $n-1$ general members of $|L|$ and write $d=L^{n}$. Then

$$
g(C) \leq\left[\frac{d-2}{N-n}\right]\left(d-N+n-1-\left(\left[\frac{d-2}{N-n}\right]-1\right) \frac{N-n}{2}\right) .
$$

Indeed $C$ is nothing but the normalization of $C^{\prime}=\varphi(C)$, so $\operatorname{deg} C^{\prime}=d$ and the inequality above is a consequence of the usual Castelnuovo's bound for the embeddings $C^{\prime} \subset \varphi(X) \subset \mathbb{P}^{N}$.

Finally, let us give the following general results we use in the sequel.

(0.10) Lemma (Nef and big degree Lemma). Let $X$ be a normal variety of dimension $n$ and let $\mathscr{L}$ be a nef and big line bundle on $X$. 
Denote by $\varphi$ the rational map associated to $|\mathscr{L}|$ and let $\#_{s}$ be the sheet number of the Stein factorization of $\varphi$. Then

$(0.10 .1) c_{1}(\mathscr{L})^{n} \geq \#_{s}(\operatorname{deg} \varphi(X))$

$(0.10 .2) c_{1}(\mathscr{L})^{n} \geq 2\left(h^{0}(\mathscr{L})-n\right)$ if $\kappa(X) \geq 0$ and $\operatorname{dim} \varphi(X)=n$.

Proof. Look at a resolution of the fundamental locus of $\varphi$

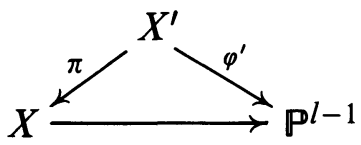

where $\mathscr{L}=h^{0}(\mathscr{L})$. Then $\mathscr{L}^{\prime}=\pi^{*} \mathscr{L} \approx \mathscr{M}+F$ where $\mathscr{M}$ is spanned and $\varphi^{\prime}$ is the morphism associated to $|\mathscr{M}|$. Further we can assume $X^{\prime}$ to be nonsingular. Take the Remmert-Stein factorization $s$ or: $X^{\prime} \rightarrow$ $Y \rightarrow \mathbb{P}^{l-1}$ of $\varphi^{\prime}$. Therefore $\mathscr{M} \approx r^{*} M$ for some ample line bundle $M$ on $Y$. Let $\#_{s}$ be the degree of $s$ and $m=\operatorname{dim} Y$. Then

$$
r^{*} c_{1}(M)^{m}=c_{1}(\mathscr{M})^{m} \text { and } c_{1}(M)^{m}[Y]=\#_{s}(\operatorname{deg} s(Y)) .
$$

If $m=n$,

$$
c_{1}\left(\mathscr{L}^{\prime}\right)^{n}=(\mathscr{M}+F) \cdot c_{1}\left(\mathscr{L}^{\prime}\right)^{n-1} \geq \mathscr{M} \cdot c_{1}\left(\mathscr{L}^{\prime}\right)^{n-1} \geq c_{1}(\mathscr{M})^{n}
$$

and $\left(^{*}\right),\left({ }^{* *}\right)$ yield $(0.10 .1)$. If $m<n$,

$$
c_{1}\left(\mathscr{L}^{\prime}\right)^{n} \geq c_{1}(\mathscr{M})^{m} \cdot c_{1}\left(\mathscr{L}^{\prime}\right)^{n-m} \geq c_{1}(\mathscr{M})^{m}
$$

and $(0.10 .1)$ follows now from $(*),(* * *)$.

As a consequence of $(0.10 .1)$ we get

$$
c_{1}(\mathscr{L})^{n} \geq \#_{s}\left(h^{0}(\mathscr{L})-n\right) .
$$

Whenever $\#_{s} \geq 2,(0.10 .2)$ is proved. If $\#_{s}=1, \varphi(X)$ has a desingularization of non-negative Kodaira dimension. It thus follows that the general surface section $S \subset \mathbb{P}^{l+1-n}$ has a desingularization of nonnegative Kodaira dimension. Now a standard argument shows that $\operatorname{deg}(S) \geq 2(N-1)=2\left(h^{0}(\mathscr{L})-n\right)$ (see also [L-S], $\left.\S 0\right)$.

(0.11) LemMa. Let $\mathscr{L}, L$ be two line bundles on an irreducible variety $X$. Assume that $L$ is spanned and big and $h^{0}(\mathscr{L}) \geq 2$. Then, given a general element $D \in|L|$, the restriction

$$
\Gamma(\mathscr{L}) \rightarrow \Gamma\left(\mathscr{L}_{D}\right)
$$

has an image of dimension $\geq 2$.

Proof. Look at the exact sequence

$$
0 \rightarrow \mathscr{L} \otimes L^{-1} \rightarrow \mathscr{L} \rightarrow \mathscr{L}_{D} \rightarrow 0
$$


If the statement is not true, then there exists a non-zero element $t \in$ $\Gamma\left(\mathscr{L} \otimes L^{-1}\right)$. Consider the restriction map

$$
\delta: \Gamma\left(\mathscr{L} \otimes L^{-1}\right) \rightarrow \Gamma\left(\mathscr{L} \otimes L_{\mid D}^{-1}\right) .
$$

Since $L$ is spanned and big we can find non-trivial $t_{1}, t_{2} \in h^{0}(L)$ whose restrictions on $D$ are not multiples of one another. If $\delta(t) \neq 0$, then $t_{1} \otimes t, t_{2} \otimes t$ are not multiples of one another on $D$ and we are done. Otherwise we would have $\delta(t) \otimes\left(t_{1}-t_{2}\right)_{D}=0$ in $\Gamma\left(l_{D}\right)$ after possibly multiplying the $t_{i}$ by non-zero constants. Since $\left(t_{1}-t_{2}\right)_{D} \neq 0$ by the above, this leads to a contradiction; here we use that $D$ is irreducible since it is general. Hence $\delta$ is the zero-map and therefore $\Gamma\left(\mathscr{L} \otimes L^{-2}\right)=\Gamma\left(\mathscr{L} \otimes L^{-1}\right) \neq(0)$. By repeating the same argument we find that $\Gamma\left(\mathscr{L} \otimes L^{-m}\right) \neq(0), m \gg 0$, again a contradiction.

For any further background material we refer to [A-S1] and [A-S2].

1. The birationality theorem. Let $L$ be an ample and spanned line bundle on a normal surface $S$. Let $\left(S^{\prime}, L^{\prime}\right)$ denote the minimal desingularization of $(S, L)$ and let $\mathscr{K}_{S}=\pi_{*} K_{S}$, be the Grauert-Riemenschneider canonical sheaf. Then the following which is essentially known ([B], [R]) can be proved.

(1.1) Theorem (Beauville, Reider). Let $(S, L),\left(S^{\prime}, L^{\prime}\right)$ be as above with $K_{S^{\prime}}+L^{\prime}$ nef and big. Further assume that $\Gamma(L)$ gives a generically one-to-one map. If $c_{1}(L)^{2} \geq 9$ then $\Gamma\left(\mathscr{K}_{S}+L\right)$ gives a birational map unless possibly $L^{\prime} \sim 3 D$, for some effective divisor $D$ with $D \cdot D=1$.

Proof. From Lemma (0.6) we see that $h^{0}\left(K_{S^{\prime}}+L^{\prime}\right)=h^{0}\left(\mathscr{K}_{S}+L\right)>0$. Hence looking at the meromorphic maps $\varphi, \psi$ associated to $\Gamma\left(\mathscr{K}_{S}+L\right)$, $\Gamma\left(K_{S^{\prime}}+L^{\prime}\right)$ respectively and from the commutative diagram

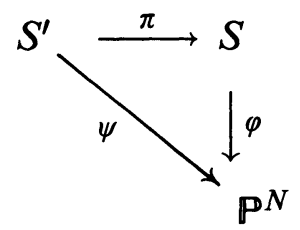

we see that it suffices to work with $\psi$ on $S^{\prime}$. To go on assume that $\psi$ is not birational. Then given a general point $x$ of $S^{\prime}$ there is a general point $y$ of $S^{\prime}$ such that the morphism

$$
\Gamma\left(K_{S^{\prime}} \otimes L^{\prime}\right) \rightarrow K_{S^{\prime}} \otimes L^{\prime} \otimes\left(\mathbb{C}_{x} \oplus \mathbb{C}_{y}\right)
$$

is not onto, where $\mathbb{C}_{x} \oplus \mathbb{C}_{y}$ is the skyscraper sheaf $\mathscr{O}_{S^{\prime}} / \mathfrak{m}_{x} \oplus \mathfrak{m}_{y}$. By Reider's theorem (0.8) there exists on $S^{\prime}$ an effective divisor $D$ passing through $x, y$ such that $L^{\prime} \cdot D=0,1$ or 2 or $L^{\prime} \cdot D=3$ and $D \cdot D=1$. 
The case $L^{\prime} \cdot D=0$ can be easily ruled out. Indeed, if $L^{\prime} \cdot D=0$, then $\pi(D)$ is a finite set. Therefore $x, y$ belong to some positive dimensional fibre of $\pi$, so that either $x$ nor $y$ is a general point; a contradiction.

The case $L^{\prime} \cdot D=3$ with $D \cdot D=1$ gives $\left(L^{\prime}-3 D\right) \cdot D=0$. Hence $L^{\prime} \sim 3 D$ or $\left(L^{\prime}-3 D\right)^{2}<0$ by the Hodge index theorem; since

$$
\left(L^{\prime}-3 D\right)^{2}=L^{\prime 2}-6 L^{\prime} \cdot D+9 D \cdot D=L^{\prime 2}-9 \geq 0
$$

it has to be $L^{\prime} \sim 3 D$.

Finally, let $L^{\prime} \cdot D=2$ or 1 . Since $L$ is ample and spanned and $\Gamma(L)$ gives a generically one-to-one map, it thus follows that $\pi(D)$ is either a smooth line or a (possibly singular) conic. Then the proper transform $D^{\prime}$ of $\pi(D)$ (or of a reduced component of $\pi(D)$ ) under $\pi$ is a nonsingular rational curve. Since $x, y$ are general points we find in this way an uncountable set of distinct nonsingular rational curves, $l$, on $S^{\prime}$. Thus $l^{2} \geq 0$. Therefore $K_{S^{\prime}} \cdot l \leq-2$ and hence $\left(K_{S^{\prime}}+L^{\prime}\right) \cdot l \leq 0$. Since $K_{S^{\prime}}+L^{\prime}$ is nef and big this leads to a contradiction by the Hodge index theorem.

(1.2) REMARK. Note that $K_{S}+L$ could also be considered to obtain an analogous result to that of the theorem above. However, the exact sequence

$$
0 \rightarrow \mathscr{K}_{S} \otimes L \rightarrow K_{S} \otimes L \rightarrow \mathscr{S} \otimes L \rightarrow 0
$$

gives an inclusion $\Gamma\left(\mathscr{K}_{S} \otimes L\right) \subset \Gamma\left(K_{S} \otimes L\right)$, so that the birational results proved for $\mathscr{K}_{S} \otimes L$ imply birationality results for the adjunction mapping associated to $\Gamma\left(K_{S}+L\right)$.

2. Some inequalities. The first two theorems we prove below generalize some results contained in [S4], $\S 3$.

The following is a consequence of Theorem 1.1 .

(2.1) Theorem. Let $L$ be a nef and big line bundle on a normal surface $S$ and let $\left(S^{\prime}, L^{\prime}\right)$ be the minimal desingularization of $(S, L)$. Suppose $K_{S^{\prime}}+L^{\prime}$ to be nef and big. Further assume $\kappa(S) \geq 0$ and let $c_{1}(L)^{2} \geq 10$ or $c_{1}(L)^{2} \geq 9$ and $L^{\prime} \nsim 3 D, D$ effective divisor with $D \cdot D=1$. Then

$$
\left(K_{S^{\prime}}+L^{\prime}\right)^{2} \geq 2\left(g(L)-q\left(S^{\prime}\right)+p_{g}\left(S^{\prime}\right)-2\right)
$$

or, equivalently,

$$
K_{S^{\prime}}^{2}+2 g(L) \geq 2\left(p_{g}\left(S^{\prime}\right)-q\left(S^{\prime}\right)\right)+d .
$$


Proof. Under the hypotheses made the map $\psi$ associated to $\Gamma\left(K_{S^{\prime}}+L^{\prime}\right)$ is birational by Theorem (1.1). Then Lemma $(0.10 .2)$ yields

$$
\left(K_{S^{\prime}}+L^{\prime}\right)^{2} \geq 2\left(h^{0}\left(K_{S^{\prime}}+L^{\prime}\right)-2\right) .
$$

Now

$$
h^{0}\left(K_{S^{\prime}}+L^{\prime}\right)=\chi\left(K_{S^{\prime}}+L^{\prime}\right)=g(L)-q\left(S^{\prime}\right)+p_{g}\left(S^{\prime}\right)
$$

so we are done.

(2.2) THEOREM. Let $L$ be a nef and big line bundle on a normal surface $S$ and let $\left(S^{\prime}, L^{\prime}\right)$ be the minimal desingularization of $(S, L)$. Assume $K_{S^{\prime}}+L^{\prime}$ to be nef and big. Then

(2.2.1) $\left(K_{S^{\prime}}+L^{\prime}\right)^{2} \geq g(L)-q\left(S^{\prime}\right)+p_{g}\left(S^{\prime}\right)-2$;

(2.2.2) $\left(K_{S^{\prime}}+L^{\prime}\right)^{2} \geq g(L)+q\left(S^{\prime}\right)-2$.

Proof. Look at the map $\psi$ associated to $\Gamma\left(K_{S^{\prime}}+L^{\prime}\right)$. Then Lemma $(0.10 .1)$ gives us

$$
\left(K_{S^{\prime}}+L^{\prime}\right)^{2} \geq \operatorname{cod} \psi\left(S^{\prime}\right)+1=h^{0}\left(K_{S^{\prime}}+L^{\prime}\right)-2
$$

and again $h^{0}\left(K_{S^{\prime}}+L^{\prime}\right)=\chi\left(K_{S^{\prime}}+L^{\prime}\right)=g(L)-q\left(S^{\prime}\right)+p_{g}\left(S^{\prime}\right)$, this leading to (2.2.1).

To prove (2.2.2) note that there exists an effective member $C^{\prime} \in$ $\left|K_{S^{\prime}}+L^{\prime}\right|$ in view of Lemma (0.6) Then the exact sequence

$$
0 \rightarrow K_{S^{\prime}} \rightarrow 2 K_{S^{\prime}} \otimes L^{\prime} \rightarrow \omega_{C^{\prime}} \rightarrow 0,
$$

where $\omega_{C^{\prime}}$ denotes the dualizing sheaf of $C^{\prime}$, gives a surjective morphism

$$
H^{0}\left(C^{\prime}, \omega_{C^{\prime}}\right) \rightarrow H^{1}\left(S^{\prime}, K_{S^{\prime}}\right) \cong H^{1}\left(S^{\prime}, \mathscr{O}_{S^{\prime}}\right)
$$

since $H^{1}\left(S^{\prime}, 2 K_{S^{\prime}}+L^{\prime}\right)=(0)$ by the Kawamata-Viehweg vanishing theorem. Now, $h^{1}(-L)=0$ since $L$ is nef and big, so that $h^{0}\left(\mathscr{O}_{C^{\prime}}\right)=$ $h^{0}\left(\mathscr{O}_{S}\right)=1$ and, hence $h^{0}\left(\omega_{C^{\prime}}\right)=h^{1}\left(\mathscr{O}_{C^{\prime}}\right)=g\left(K_{S^{\prime}}+L^{\prime}\right)$. Therefore

$$
g\left(K_{S^{\prime}}+L^{\prime}\right) \geq q\left(S^{\prime}\right)
$$

so by the genus formula we find

$$
\begin{aligned}
2 q\left(S^{\prime}\right)-2 & \leq 2 g\left(K_{S^{\prime}}+L^{\prime}\right)-2=\left(2 K_{S^{\prime}}+L^{\prime}\right) \cdot\left(K_{S^{\prime}}+L^{\prime}\right) \\
& =2\left(K_{S^{\prime}}+L^{\prime}\right)^{2}-L^{\prime} \cdot\left(K_{S^{\prime}}+L^{\prime}\right),
\end{aligned}
$$

that is

$$
\left(K_{S^{\prime}}+L^{\prime}\right)^{2} \geq g(L)+q\left(S^{\prime}\right)-2
$$


(2.3) Corollary. Let $(S, L),\left(S^{\prime}, L^{\prime}\right)$ be as in Theorem (2.2) and let $d=L \cdot$ L. Further assume that $q\left(S^{\prime}\right)>0$ and $p_{g}\left(S^{\prime}\right)=0$. Then

$$
g(L) \geq d / 3+3 q\left(S^{\prime}\right)-2 .
$$

Proof. From [S1], (0.8.2) we know that $K_{S^{\prime}} \cdot K_{S^{\prime}} \leq 8\left(1-q\left(S^{\prime}\right)\right)$ and the genus formula reads

$$
\left(K_{S^{\prime}}+L^{\prime}\right)^{2}+d=K_{S^{\prime}} \cdot K_{S^{\prime}}+4 g(L)-4 .
$$

Hence

$$
8\left(1-q\left(S^{\prime}\right)\right) \geq\left(K_{S^{\prime}}+L^{\prime}\right)^{2}+d-4 g(L)+4 .
$$

By combining the inequality above with $(2.2 .2)$ we get the result.

(2.4) REMARK. Note that whenever $S^{\prime}$ is birationally ruled and $\left|L^{\prime}\right|$ contains a smooth curve $C$ which meets a general fibre of the ruling $S^{\prime} \rightarrow R, R$ nonsingular curve, in $t$ points, then the Hurwitz theorem gives us

$$
g(L) \geq 1+t\left(q\left(S^{\prime}\right)-1\right) .
$$

However, such an inequality is usually weaker than (2.3) for $t$ around 3.

(2.5) Corollary. Let $(S, L),\left(S^{\prime}, L^{\prime}\right)$ be as in Corollary (2.3). Further assume $g(L) \leq 6$. Then $q\left(S^{\prime}\right) \leq 2$. If $q\left(S^{\prime}\right)=2$, then either $g(L)=5$ with $d \leq 3$ or $g(L)=6$ with $d \leq 6$. Furthermore if $L$ is spanned and $\Gamma(L)$ gives a generically one-to-one map then $q\left(S^{\prime}\right)=2$ implies that $h^{0}(L)=4, g(L)=6$ and $d=5$ or 6 .

Proof. Indeed $q\left(S^{\prime}\right) \geq 3$ implies $g(L)>7$ by (2.3) above, so that $q\left(S^{\prime}\right) \leq 2$. Again (2.3) and $q\left(S^{\prime}\right)=2$ imply $g(L)=5$ or 6 with the stated bound for $d$.

If $L$ is spanned and $\Gamma(L)$ gives a generically one-to-one map then the Castelnuovo's bound (0.9) shows $d \geq 4$ if $g(L)=5$. Therefore $g(L)=6$. By combining (2.3) and (0.9) we find $d=5$ or 6 and $h^{0}\left(L^{\prime}\right)=h^{0}(L)=4$.

Finally let us give an easy but useful generalization of some of Sommese's results contained in [S3].

(2.6) Theorem. Let $X$ be an irreducible variety of dimension $n$. Let $L$ be a spanned and big line bundle on $X$. Let $S \subset X$ be a general surface section obtained as transversal intersection of $n-2$ general 
members of $|L|$ and let $\left(S^{\prime}, L^{\prime}\right)$ be the minimal desingularization of $(S, L)$. If $\kappa(X) \geq 0$ then one has
(2.6.1) $K_{S^{\prime}} \cdot K_{S^{\prime}} \geq(n-3) K_{S^{\prime}} \cdot L_{S^{\prime}}^{\prime}+(n-2) L_{S^{\prime}}^{\prime} \cdot L_{S^{\prime}}^{\prime}$
(2.6.2) $K_{S^{\prime}} \cdot L_{S^{\prime}}^{\prime} \geq(n-2) L_{S^{\prime}}^{\prime} \cdot L_{S^{\prime}}^{\prime}$

Note. $K_{S} \cdot L_{S}=K_{S^{\prime}} \cdot L_{S^{\prime}}^{\prime}$ and $L_{S^{\prime}}^{\prime} \cdot L_{S^{\prime}}^{\prime}=L_{S} \cdot L_{S}$. Furthermore if either inequality is an equality then $\kappa(X)=0$.

Proof. By using Bertini's type theorems and the fact that $S$ is general one sees that there exists a commutative diagram

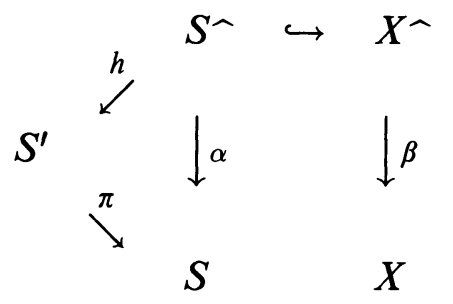

where $\alpha, \beta$ are desingularizations, $S^{\wedge}=\beta^{-1}(S), \alpha=\beta_{\mid S^{\wedge}}$ and it factorizes through some morphism $h$ since $\pi$ is the minimal desingularization of $S$. Note that by hypothesis $K_{X^{\wedge}}$ is $\mathbb{Q}$-effective. Note also that $\kappa\left(S^{\prime}\right)=2$ since $\kappa(X) \geq 0$, and hence $K_{S^{\prime}}+L^{\prime}$ is nef and big by [A-S1]. Let $L^{\wedge}=\beta^{*} L$. From the commutativity of the diagram it thus follows that $L_{\hat{S}^{\wedge}} \approx h^{*} L_{S^{\prime}}^{\prime}$. Then we can compute:

$$
\begin{aligned}
0 & \leq h^{*}\left(K_{S^{\prime}}+L_{S^{\prime}}^{\prime}\right) \cdot\left(K_{X^{\wedge}} \cdot L^{\wedge n-2}\right) \\
& =h^{*}\left(K_{S^{\prime}}+L_{S^{\prime}}^{\prime}\right) \cdot\left(K_{S^{\wedge}}-(n-2) L_{S^{\wedge}}\right) \\
& =\left(K_{S^{\prime}}+L_{S^{\prime}}^{\prime}\right) \cdot K_{S^{\prime}}-(n-2)\left(K_{S^{\prime}}+L_{S^{\prime}}^{\prime}\right) \cdot L_{S^{\prime}}^{\prime} \\
& =K_{S^{\prime}} \cdot K_{S^{\prime}}-(n-2) L_{S^{\prime}}^{\prime} \cdot L_{S^{\prime}}^{\prime}-(n-3) K_{S^{\prime}} \cdot L_{S^{\prime}}^{\prime}
\end{aligned}
$$

which leads to (2.6.1). Similarly one has

$$
\begin{aligned}
K_{S^{\prime}} \cdot L_{S^{\prime}}^{\prime} & =h^{*} K_{S^{\prime}} \cdot L_{S^{\wedge}}=K_{S^{\wedge}} \cdot L_{S^{\wedge}} \\
& =\left(K_{X^{\wedge} \mid S^{\wedge}}+(n-2) L L_{S^{\wedge}}\right) \cdot L_{S^{\wedge}} \geq(n-2) L_{\widehat{S^{\wedge}}} \cdot L_{\hat{S^{\wedge}}}
\end{aligned}
$$

that is (2.6.2). To prove the last part of the statement, note that the equality in (2.6.1) or (2.6.2) gives respectively

$$
K_{X^{\wedge} \mid S^{\wedge}} \cdot h^{*}\left(K_{S^{\prime}}+L_{S^{\prime}}^{\prime}\right)=0
$$

or

$$
K_{X^{\wedge} \mid S^{\wedge}} \cdot L_{\hat{S}^{\wedge}}=0 .
$$

Now if $h^{0}\left(K_{X^{\wedge}}^{N}\right) \geq 2$ for some $N \geq 1$, then $h^{0}\left(\left(K_{X^{\wedge} \mid S^{\wedge}}\right)^{N}\right) \geq 2$ by Lemma (0.11). Therefore since $h^{*}\left(K_{S^{\prime}}+L_{S^{\prime}}^{\prime}\right)$ and $L_{S^{\prime}}^{\prime}$ are nef and big 
a straightforward check shows that the intersection numbers $K_{X^{\wedge} \mid S^{\wedge}}$. $h^{*}\left(K_{S^{\prime}}+L_{S^{\prime}}^{\prime}\right)$ and $K_{X^{\wedge} \mid S^{\wedge}} \cdot L_{S^{\wedge}}$ must be positive. It thus follows that $h^{0}\left(K_{X^{\wedge}}^{N}\right) \leq 1$ for all $N>0$, whence $\kappa(X)=0$.

The following consequence of the theorem above is a slight generalization of $(0.5 .1)$ in [L-S].

(2.7) Corollary. Let $(X, L)$ be as in Theorem (2.6) and let $d=$ $L^{\cdot N}$. Then

$$
d \leq \frac{2(g(L)-1)}{n-1}
$$

with equality only if $\kappa(X)=0$.

Proof. From (2.6.2) and the genus formula we get

$$
2 g(L)-2=K_{S^{\prime}} \cdot K_{S^{\prime}}+L_{S^{\prime}}^{\prime} \cdot L_{S^{\prime}}^{\prime} \geq(n-1) L_{S^{\prime}}^{\prime} \cdot L_{S^{\prime}}^{\prime}=(n-1) d .
$$

3. An application to hyperelliptic hyperplane sections. First of all note that it is equivalent to consider pairs $(S, L)$ where $S$ is a normal surface with $L$ an ample spanned line bundle such that $\Gamma(L)$ gives a generically one-to-one map and pairs $(S, L)$ where $S$ is the normalization $\eta: S \rightarrow \Sigma$ of an irreducible surface $\Sigma \subset \mathbb{P}^{r}$ and $L \approx \eta^{*} \mathscr{O}(1)$. Indeed, $\eta^{*} \mathscr{O}(1)$ is ample and spanned and $\Gamma\left(\eta^{*} \mathscr{O}(1)\right)$ gives a generically one-to-one map.

Now let $\left(S^{\prime}, L^{\prime}\right)$ be the minimal desingularization of a pair $(S, L)$ as above. The following is the analogue of a result of Sommese working in the case when $q\left(S^{\prime}\right)=0$ (see [S1], §4) and of a result of Van de Ven where $L$ has to verify the two extra conditions $h^{0}(L) \geq 7$ and $L \cdot L \geq 10$ (see [V], Cor. IV).

(3.1) THEOREM. With the notation as above, let $L$ be an ample and spanned line bundle on a normal surface $S$. Further assume that $\Gamma(L)$ gives a generically one-to-one map and $c_{1}(L)^{2} \geq 10$ or $c_{1}(L)^{2} \geq 9$ and $L^{\prime} \nsim 3 D, D$ effective divisor with $D \cdot D=1$. If there exists a smooth hyperelliptic curve $C \in|L|$ then $p_{g}\left(S^{\prime}\right)=0, d=c_{1}(L)^{2} \geq g(L)+2$ and either

(3.1.1) $q\left(S^{\prime}\right)>0, h^{0}(L)=4, g(L)+2 \geq 3 q\left(S^{\prime}\right)+d / 3$ and there exist at most finitely many smooth hyperelliptic curves in $|L|$;

(3.1.2) $(S, L)$ is a cone or a scroll; or

(3.1.3) $\left(S^{\prime}, L^{\prime}\right)$ is a conic bundle over a smooth curve.

Proof. Let $C$ be a nonsingular hyperelliptic curve belonging to $|L|$. It should be noted that $C^{\prime}=\pi^{-1}(C)$ is a nonsingular hyperelliptic 
curve in $\left|L^{\prime}\right|$ since $C$ does not pass through the singular points of $S$; vice versa, given any smooth hyperelliptic curve $C^{\prime} \in\left|L^{\prime}\right|, \pi\left(C^{\prime}\right)=C$ is an hyperelliptic curve in $|L|$ since $L^{\prime}=\pi^{*} L$.

From now on, we can assume that $K_{S^{\prime}}+L^{\prime}$ is nef and big. Otherwise in view of [A-S1], (2.5), (2.7), $\left(S^{\prime}, L^{\prime}\right)$ is either a conic bundle or a scroll over a nonsingular curve or the minimal desingularization of a quadric cone. Now an easy argument shows that if $(S, L) \neq\left(S^{\prime}, L^{\prime}\right)$ and $\left(S^{\prime}, L^{\prime}\right)$ is a scroll then $(S, L)$ is a cone. Thus we fall in one of classes (3.1.2) or (3.1.3).

First, note the fact that there exist at most finitely many smooth hyperelliptic curves $C$ in $|L|$ is clear. Otherwise, if $C^{\prime}=\pi^{-1}(C)$, $K_{C^{\prime}} \approx\left(K_{S^{\prime}}+L^{\prime}\right)_{\mid C^{\prime}}$ and hence the map associated to $\Gamma\left(K_{S^{\prime}}+L^{\prime}\right)$ would be at least 2 to 1 on a dense set of curves, this contradicting Theorem 1.1. To go on, we need the following

Claim. Let $x \in C$ be a ramification point for the canonical map associated to $\Gamma\left(K_{C}\right)$. If $q\left(S^{\prime}\right)=0$ and a smooth $C^{\prime} \in\left|L^{\prime}-x\right|$ is tangent to $C$ at $x$ or if $q\left(S^{\prime}\right)>0$ and a smooth $C^{\prime} \in\left|L^{\prime}-x\right|$ is tangent to $C$ at $x$ of the 2 nd order, then $C^{\prime}$ is hyperelliptic.

Proof of the Claim. Note that the proof in [S2], (4.2) works with almost no change to give the $q\left(S^{\prime}\right)>0$ result. We give here the proof of the stronger statement when $q\left(S^{\prime}\right)=0$. Take an element $A \in$ $\left|K_{S^{\prime}}+L^{\prime}-x\right|$. Then the local intersection multiplicity $(A \cdot C)_{x}$ at $x$ is nothing but the zero's order of a 1-form belonging to $\Gamma\left(K_{C}\right)$, therefore $(A \cdot C)_{x} \geq 2$. It thus follows that $\left(A \cdot C^{\prime}\right)_{x} \geq 2$ also. Indeed, if $\left(A \cdot C^{\prime}\right)_{x}=1$ then $A$ would be smooth at $x$ and transverse to $C^{\prime}$ at $x$ and hence to any smooth curve $C$ tangent to $C^{\prime}$ at $x$. Thus, since the map

$$
\Gamma\left(K_{S^{\prime}}+L^{\prime}\right) \rightarrow \Gamma\left(K_{C^{\prime}}\right) \rightarrow 0
$$

is onto $q\left(S^{\prime}\right)$ being zero, we see that any 1-form $\omega \in \Gamma\left(K_{C^{\prime}}\right)$ which vanishes at $x$, vanishes to the 2 nd order at $x$. This means that $C^{\prime}$ is hyperelliptic (see again [S2]).

From the claim we infer that if $q\left(S^{\prime}\right)=0$ and $h^{0}\left(L^{\prime}\right)=4$ or $q\left(S^{\prime}\right)>0$ and $h^{0}\left(L^{\prime}\right) \geq 5$ there is a pencil of smooth hyperelliptic curves $C^{\prime} \in\left|L^{\prime}\right|$ on $S^{\prime}$. Again, looking at the restriction $\left(K_{S^{\prime}}+L^{\prime}\right)_{\mid C^{\prime}} \approx K_{C^{\prime}}$ the same argument as above leads to a contradiction in view of Theorem 1.1. Note $h^{0}(L) \geq 4$ since $h^{0}(L)=3$ would imply $(S, L) \cong\left(\mathbb{P}^{2}, \mathscr{O}(1)\right)$, by Zariski's Main Theorem, contradicting $L \cdot L \geq 9$. Thus it has to be 
$q\left(S^{\prime}\right)>0$ and $h^{0}(L)=4$. To prove that $p_{g}\left(S^{\prime}\right)=0$ the same argument as in [S1], (0.8.3) works.

Moreover, $h^{0}\left(L_{C}\right) \geq 3$ because $h^{0}(L) \geq 4$. Therefore $\chi\left(L_{C}\right)=$ $d-g(L)+1 \geq 3$ since $h^{1}\left(L_{C}\right)=0$, which gives $d \geq g(L)+2$.

Finally we apply Corollary (2.3) to get $g(L)+2 \geq d / 3+3 q\left(S^{\prime}\right)$ whenever $q\left(S^{\prime}\right)>0$ and this completes the proof.

(3.2) REMARK. It is worth noting that case (3.1.1) in Theorem (3.1) above does not occur whenever $S$ is smooth. Indeed, let $C \in|L|$ be a smooth hyperelliptic curve and $p: C \rightarrow \mathbb{P}^{1}$ the associated 2 to 1 covering. For a general point $z \in \mathbb{P}^{1}$, let $p^{-1}(z)=P+Q$. Then $\varphi(P)=\varphi(Q)$ where $\varphi$ denotes the map associated to $\Gamma\left(K_{S}+L\right)$, so that [R], $\S 1$ applies to say that there exists an effective divisor $D_{z}$ on $S, D_{z} \supset p^{-1}(z)$ such that either $L \cdot D_{z}=1, D_{z} \cdot D_{z}=-1$ or 0 or $L \cdot D_{z}=2, D_{z} \cdot D_{z}=0$.

Let $L \cdot D_{z}=1$. Note that the case $D_{z} \cdot D_{z}=-1$ is ruled out since the $D_{z}$ 's are parametrized by $\mathbb{P}^{1}$, so $D_{z} \cdot D_{z}=0$, and $(S, L)$ is a scroll as in case (3.1.2).

If $L \cdot D_{z}=2, D_{z} \cdot D_{z}=0$, either $D_{z}$ is irreducible or not. If it is not irreducible, the general $D_{z}$ consists of 2 lines, one of which, say $\ell$, moves. Using the Hodge index theorem and the assumption $c_{1}(L)^{2} \geq 10$ we conclude that $l \cdot l=0$ on $S$, hence we fall again in the scroll case. If $D_{z}$ is irreducible, then $D_{z_{1}}$ meets $D_{z_{2}}$ for general $z_{1}, z_{2}$ in $\mathbb{P}^{1}$ in $D_{z_{1}} \cdot D_{z_{2}}=0$ points. Therefore since the parameter space $\mathbb{P}^{1}$ is rational the two irreducible curves $D_{z_{1}}, D_{z_{2}}$ are linearly equivalent. Thus we have two disjoint divisors giving the same line bundle. So the line bundle is spanned and by Bertini, the general $D_{z}$ is smooth. Furthermore, since $L \cdot D_{z}=2$, either $D_{z}$ is a rational curve, and $(S, L)$ is a conic bundle as in case (3.1.3), or the map, associated to $\Gamma(L)$ is 2 to 1 on the $D_{z}$ 's. This contradicts the assumption that $\Gamma(L)$ gives a generically 1-to-1 map, and therefore we are done.

\section{REFERENCES}

[A-S1] M. Andreatta and A. J. Sommese, Generically ample divisors on normal Gorenstein surfaces, to appear in Contemporary Mathematics, Proc. Iowa City Singularities Conference, 1986.

[A-S2] $\longrightarrow$, On the adjunction mapping for singular projective varieties, Forum Math. J., 1 (1989), 143-152.

[B] A. Beauville, Letter on Reider's method, on March 17th 1986.

[E] L. Ein, Surfaces with a hyperelliptic hyperplane section, Duke Math. J., 50 (1983), 685-694. 
[L-S] E. L. Livorni and A. J. Sommese, Threefolds of Non Negative Kodaira Dimension with Sectional Genus Less than or Equal to 15, Annali Scuola Normale Superiore, Pisa, XIII, 4 (1986), 537-557.

[R] I. Reider, Vector bundles of rank two and linear systems on algebraic surfaces, Ann. of Math., 127 (1988), 309-316.

[Se] F. Serrano, The adjunction mapping and hyperelliptic divisors on a surface, J. Reine Angew. Math., 381 (1987), 50-109.

[S1] A. J. Sommese, Hyperplane sections of projective surfaces I-The Adjunction Mapping, Duke Math. J., 46 (1979), 377-401.

[S2] _ Hyperplane Sections, Proceedings of the Algebraic Geometry Conference, University of Illinois at Chicago Circle, 1980, Lect. Notes in Math., 862, Springer (1981).

[S3] _ On the minimality of hyperplane sections of projective threefolds. J. Reine Angew. Math., 329 (1981), 16-41.

[S4] _ Ample divisors on Gorenstein varieties, Proceedings on Complex Geometry Conference, Nancy 1985, Revue de l'Institut E. Cartan, 10 (1986).

[S5] _ - On the Adjunction Theoretic Structure of Projective Varieties, Complex Analysis and Algebraic Geometry, Proceedings, Göttingen 1985, Springer Lecture Notes 1194, (1986), 175-213.

[S-V] A. J. Sommese and A. Van de Ven, On the adjunction mapping, Math. Ann., 278 (1987), 593-603.

[V] A. Van de Ven, On the 2-connectedness of very ample divisors on a surface, Duke Math. J., 46 (1979), 403-407.

Received January 25, 1988. We would like to express our thanks to the Max-PlanckInstitut für Mathematik for making this joint work possible. The third author would also like to thank the University of Notre Dame and the National Science Foundation (DMS 87-22330) for their support.

UNIVERSITY OF TRENTO

38100 TRENTO (ItAly)

UNIVERSITY OF GENOVA

Via L. B. Alberti 4

16132 Genova (ItAly)

AND

UNIVERSITY OF NOTRE DAME

Notre Dame, IN 46556 (USA). 



\section{PACIFIC JOURNAL OF MATHEMATICS EDITORS}

\author{
V. S. VARADARAJAN \\ (Managing Editor) \\ University of California \\ Los Angeles, CA 90024-1555-05 \\ Herbert Clemens \\ University of Utah \\ Salt Lake City, UT 84112 \\ ThOMAS ENRIGHT \\ University of California, San Diego \\ La Jolla, CA 92093
}

\section{R. FINN}

Stanford University

Stanford, CA 94305

HeRmann FlaschKa

University of Arizona

Tucson, AZ 85721

VAUGHAN F. R. JONES

University of California

Berkeley, CA 94720

Steven KerckhofF

Stanford University

Stanford, CA 94305

\author{
RobION KIRBY \\ University of California \\ Berkeley, CA 94720 \\ C. C. Moore \\ University of California \\ Berkeley, CA 94720 \\ HAROLD STARK \\ University of California, San Diego \\ La Jolla, CA 92093
}

\section{ASSOCIATE EDITORS}
R. ARENS
E. F. BECKenBach
B. H. NeumanN
F. WOLF
K. YoshidA (1906-1982)
(1904-1989)

\section{SUPPORTING INSTITUTIONS}

UNIVERSITY OF ARIZONA

UNIVERSITY OF BRITISH COLUMBIA

CALIFORNIA INSTITUTE OF TECHNOLOGY

UNIVERSITY OF CALIFORNIA

MONTANA STATE UNIVERSITY

UNIVERSITY OF NEVADA, RENO

NEW MEXICO STATE UNIVERSITY

OREGON STATE UNIVERSITY
UNIVERSITY OF OREGON

UNIVERSITY OF SOUTHERN CALIFORNIA

STANFORD UNIVERSITY

UNIVERSITY OF HAWAII

UNIVERSITY OF TOKYO

UNIVERSITY OF UTAH

WASHINGTON STATE UNIVERSITY

UNIVERSITY OF WASHINGTON 


\section{Pacific Journal of Mathematics}

Vol. 142, No. $1 \quad$ January, 1990

Marco Andreatta, Mauro Beltrametti and Andrew Sommese, Generic properties of the adjuction mapping for singular surfaces and applications

Chen-Lian Chuang and Pjek-Hwee Lee, On regular subdirect products of

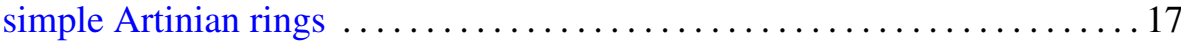

Fernando Giménez and Vicente Miquel Molina, Volume estimates for real hypersurfaces of a Kaehler manifold with strictly positive holomorphic sectional and antiholomorphic Ricci curvatures $\ldots \ldots \ldots \ldots \ldots \ldots 23$

Richard J. Griego and Andrzej Korzeniowski, Asymptotics for certain Wiener integrals associated with higher order differential operators

Abdeslam Mesnaoui, Unitary bordism of classifying spaces of quaternion

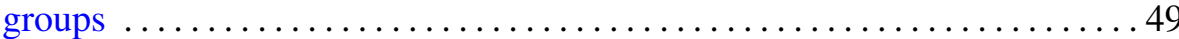

Abdeslam Mesnaoui, Unitary cobordism of classifying spaces of quaternion

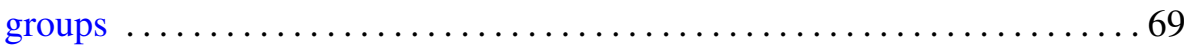

Jesper M. Møller, On equivariant function spaces $\ldots \ldots \ldots \ldots \ldots \ldots \ldots \ldots$

Bassam Nassrallah, A $q$-analogue of Appell's $F_{1}$ function, its integral representation and transformations

Peter A Ohring, Solvability of invariant differential operators on metabelian

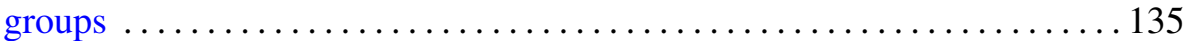

Athanase Papadopoulos and R. C. Penner, Enumerating pseudo-Anosov foliations

Ti-Jun Xiao and Liang Jin, On complete second order linear differential

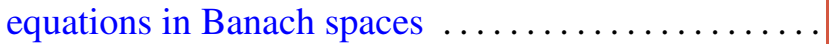

Carl Widland and Robert F. Lax, Weierstrass points on Gorenstein curves 